\title{
Enjeux de l'immigration féminine en Irlande au XXI siècle et politiques gouvernementales
}

Marie-Jeanne Da Col Richert

\section{(2) OpenEdition \\ 1 Journals}

Édition électronique

URL : https://journals.openedition.org/etudesirlandaises/4473

DOI : $10.4000 /$ etudesirlandaises. 4473

ISSN : 2259-8863

Éditeur

Presses universitaires de Caen

Édition imprimée

Date de publication : 30 juin 2015

Pagination : 119-131

ISBN : 978-2-7535-4082-8

ISSN : 0183-973X

Référence électronique

Marie-Jeanne Da Col Richert, «Enjeux de l'immigration féminine en Irlande au xxl ${ }^{e}$ siècle et politiques gouvernementales », Études irlandaises [En ligne], 40-1 | 2015, mis en ligne le 30 juin 2017, consulté le 20 avril 2022. URL : http://journals.openedition.org/etudesirlandaises/4473 ; DOI : https://doi.org/ 10.4000/etudesirlandaises.4473

\section{(c) (i) (8) (-)}

Études irlandaises est mise à disposition selon les termes de la Licence Creative Commons Attribution - Pas d'Utilisation Commerciale - Partage dans les Mêmes Conditions 4.0 International. 


\title{
Enjeux de l'immigration féminine en Irlande au $\mathrm{XXI}^{\mathrm{e}}$ siècle et politiques gouvernementales
}

\author{
Marie-Jeanne DA Col RicherT \\ Université de Strasbourg
}

\section{Résumé}

Les migrantes en Irlande au $\mathrm{XxI}^{\mathrm{e}}$ siècle souffrent de discrimination en tant que femmes dans une société patriarcale. Elles sont aussi confrontées à des difficultés comme employées au statut précaire, recrutées souvent au-dessous de leurs qualifications. Le travail domestique dans l'économie grise bafoue leurs droits. La législation sur l'immigration, complexe, non dénuée d'arbitraire, les pénalise. Pour démanteler les réseaux de prostitution dont nombre de migrantes sont victimes, une campagne abolitionniste prend corps en Irlande.

Mots clés : immigration, femmes - représentation politique, Irlande - politiques économiques et sociales, minorités ethniques

\section{Abstract}

Twenty-first century female migrants in Ireland are not only discriminated against as women in a patriarchal society, but also as foreigners whose status is precarious and whose jobs are often below their qualifications. For those who are relegated to the grey economy living conditions are particularly harsh. Obstacles to female migrants' integration result from complex immigration policies, whose enforcement is not always immune from arbitrariness. Mobilization in Ireland against prostitution networks whose victims are female migrants has allowed an abolitionist campaign to develop lately.

Keywords : immigration, women - political representation, Ireland - socio-economic policies, ethnic minorities

L'Irlande est devenue un lieu de destination pour les migrants à une période très récente. La prospérité économique de la fin des années 90, désignée par Tigre celtique, a incité le gouvernement à ouvrir les frontières aux travailleurs étrangers. Pendant une dizaine d'années, des flux de personnes en quête d'emplois sont arrivés dans le pays, notamment en 2004, puis en 2007, lors de l'accession de nouveaux états membres à l'Union européenne ${ }^{1}$. Alors que les nouveaux venus de la décennie du Tigre celtique étaient des hommes surtout au début, attirés par

1. Steven Loyal, Understanding Immigration in Ireland, Manchester University Press, 2011, p. 4. 
le secteur de la construction, les proportions se sont inversées ensuite ${ }^{2}$, les femmes formant une majorité. À partir de données aussi récentes que possible, la première partie analyse la place de la main-d'œuvre féminine étrangère dans le monde de l'emploi notamment, compte tenu des réformes successives qui ont affecté les droits des différentes catégories d'immigrantes durant les deux dernières décennies. La deuxième partie du travail s'applique à étudier la condition des immigrantes dans la société irlandaise en dégageant des constantes. Qu'en est-il du statut et du rôle de cette catégorie de femmes en termes d'émancipation, d'intégration, d'aliénation? Dans un troisième temps, les initiatives destinées à lutter contre les aspects les plus sombres de l'immigration féminine sont présentées. Ce travail vise à une mise en perspective de l'Irlande comme pays d'accueil pour les diverses catégories de femmes migrantes.

\section{圈 Discrimination}

L'émergence du succès économique, favorisé par la dérégulation et la flexibilité inhérentes à la mondialisation du marché ${ }^{3}$ au milieu des années 1990, a attiré des étrangers en quête d'emplois, et entrâné de nombreux retours d'Irlandais établis à l'étranger. Jane Pillinger ${ }^{4}$, universitaire, à présent chercheuse indépendante, membre de l'Immigrant Council of Ireland, donne les caractéristiques de l'immigration féminine dans ses travaux récents et en souligne les contrastes : à l'époque actuelle les immigrantes sont aussi bien des femmes diplômées et qualifiées que des personnes pauvres, dépourvues d'atouts sur le marché de l'emploi. Elles sont de plus en plus nombreuses à se déplacer seules, notamment pour subvenir aux besoins de leurs enfants restés au pays 5 . Mais l'étude de l'immigration féminine en Irlande se heurte à un obstacle majeur : les données objectives et les statistiques établies dans une perspective hommes/femmes font défaut. En outre l'échange des données entre les services et agences gouvernementaux s'opère mal ${ }^{6}$. Les chiffres utilisés pour ce travail sont donc obtenus en croisant ceux qui concernent l'ensemble des immigrants en Irlande avec ceux qui documentent la question hommes/femmes. Des lignes générales, mises en relief dans les rapports officiels et travaux des chercheurs, servent aussi de point d'appui à l'argumentation.

\footnotetext{
2. Central Statistics Office (CSO), Women and Men in Ireland 2008, Government Publications Sales Office (GPSO), Dublin, 2009, p. 24.

3. Loyal, 2011, op. cit., p. 2 et 14.

4. Jane Pillinger, The Feminisation of Migration, Immigrant Council of Ireland (ICI), Dublin, 2007; Jane Pillinger, "The Changing Face of Ireland, Gender and the Feminisation of Migration ", dans Ursula Barry (ed.), Where are we now?, Dublin, Tasc at New Island, 2008, p. 184-215.

5. Pillinger, op. cit., 2008, p. 184.

6. Ibid., 2007, p. 17-18.
} 
Une perspective générale sur l'immigration indique $10 \%$ d'étrangers en Irlande, en $2006^{7}$, à la veille de la crise. Initialement, le nombre de femmes approchait celui des hommes. En 2008, en raison notamment du déclin du secteur de la construction, elles étaient devenues plus nombreuses ${ }^{8}$. L'année record pour le nombre d'immigrantes est 2006, avec plus de 50000 arrivantes, qui représentaient alors environ $49 \%$ du total ${ }^{9}$. Le recensement de 2011 révèle que la crise n’a pas tari le flux : le nombre d'immigrants était alors proche de 545000 , soit $12 \%$ de la population (un tiers de plus environ, en pourcentage, qu'en 2006). Comme au début de la crise, les femmes étaient un peu plus nombreuses que les hommes.

Peut-on dégager un profil général de cette population féminine? Les études de ces quinze dernières années montrent qu'une majorité des immigrants appartient à l'Union Européenne. Quant aux immigrants venus du reste du monde, ils forment un pourcentage stable de $25 \%$, si l'on inclut les Américains ${ }^{10}$. Il est raisonnable de penser que ces statistiques, qui ne dissocient pas hommes et femmes, constituent des données de référence à la fois pour l'un et l'autre sexe. En effet, le nombre d'hommes et femmes est sensiblement équivalent dans la population immigrante, dont la grande majorité se situe dans la tranche d'âge 20/40 ans ${ }^{11}$, ce qui en fait d'emblée des candidats à l'emploi, ou immigrants économiques. Le taux d'emploi des femmes migrantes est proche de celui des Irlandaises : $57 \%$ pour les premières en $2009,56,6 \%$ pour les secondes ${ }^{12}$, alors même que la crise s'installait durablement.

Qu'en est-il des secteurs de l'emploi où les migrants sont recrutés? Les différences traditionnelles entre métiers féminins et masculins sont marquées ${ }^{13}$; si les hommes ont été massivement recrutés dans le secteur de la construction, les femmes se sont généralement dirigées vers des emplois dans le secteur de la santé et du travail social, féminisé à $80 \%{ }^{14}$. Les immigrantes des pays hors Europe représentent une proportion significative de ce personnel ${ }^{15}$ : le pourcentage est passé de $2 \%$ en 1998 à $16,5 \%$ en $2006^{16}$. Entre 2000 et 2008, certains hôpitaux

7. Philip O'Connell et Frances McGinnity, Immigrant at Work, Ethnicity and Nationality in the Irish Labour Market, Equality Authority and ESRI, Dublin, 2008, p. 1.

8. Women and Men in Ireland 2008, CSO, Dublin, 2008, p. 24.

9. Women and Men in Ireland 2011, CSO Cork, p. 15; [www.cso.ie], consulté le 13 août 2013.

10. Africains, Nigérians avant tout : $8 \%$ de ce sous-total; Asiatiques : $11 \%$, Chinois, Indiens, Philippins notamment, Migration Nation: Statement on Integration Strategy and Diversity Management, Office of the Minister for Integration, May 2008.

11. CSO, Non-Irish Nationals living in Ireland, GPSO, Dublin, 2008, p. 15.

12. ESRI, Annual Monitoring Report On Integration (AMRI) 2010, p. 17.

13. Ursula Barry, Short Report on Migrant Women in Ireland (part B), UCD School of Social Justice Working papers Series Number 10(4): 1-10, oct. 2010, p. 3

14. W\&M in Ir. 2011, p. 19.

15. Annual Monitoring Record on Integration (AMRI) 2010, ESRI, Dublin, avril 2011 p. 41.

16. ESRI, Projecting the Future Numbers of Migrant Workers in the Health and Social care Sectors in Ireland, Working paper no. 275, Jan. 2009, p. 10. 
dublinois comptaient de 50 à $80 \%$ d'infirmières étrangères hors Europe, notamment indiennes et philippines ${ }^{17}$. Une forte proportion, environ $20 \%$, des immigrantes de l'Europe des 25 et $27^{18}$, ont, en revanche, été embauchées dans certains secteurs des services telle la vente de détail, où les emplois se sont multipliés durant le Tigre celtique. Dans l'hôtellerie et la restauration, la main-d'œuvre est féminine à $59 \%$ et compte plus de $35 \%$ d'immigrants en 2008, dont une majorité en provenance des nouveaux États membres ${ }^{19}$ de l'UE.

Dans l'ensemble des secteurs de l'emploi caractérisés par une forte présence de main-d'œuvre féminine étrangère, des problèmes de discrimination sont dénoncé $^{20}$. Des travaux de recherche qualitative mettent en perspective les situations vécues par des employées immigrantes dans les structures d'accueil pour personnes âgées. Les infirmières et aide-soignantes indiennes et philippines, mais aussi polonaises, évoquent la ségrégation et les humiliations, et dénoncent des inégalités de traitement ${ }^{21}$.

L'inspection du travail (National Employment Rights Authority) fait état de nombreuses violations de la législation dans la restauration et l'hôtellerie; les plaintes d'employés exploités sont nombreuses, $60 \%$ se disent maltraités, mais renoncent à se défendre par crainte de perdre leur emploi ${ }^{22}$. Les résultats d'une enquête, publiés par l'ESRI en 2006, mettent en perspective ce problème social, qui concerne les migrants dans l'ensemble ${ }^{23}$. Quant aux mauvais traitements, insultes et autres formes de harcèlement répétés, ils sont dénoncés par ceux qui sont originaires de pays extérieurs à la zone Europe ${ }^{24}$. Une étude quantitative de 2008, menée par l'ESRI et l'Equality Authority 25, qui élargit l'enquête de 2006, et prend en compte le critère hommes/femmes, confirme ces constats. Toutefois, en matière d'accès à l'emploi, les hommes sont, semble-t-il, plus souvent victimes de discrimination que les femmes ${ }^{26}$.

17. Niamh Humphries et al., " "I won't be staying here for long”: a qualitative study on the retention of migrant nurses in Ireland ", Human Resources for Health 2009, 7, p. 68, Dublin, août 2009, p. 3.

18. 10 nouveaux Etats membres en 2004 et 2 en 2007.

19. Migrants Rights Centre of Ireland (MRCI)'s Restaurant Workers Action Group (RWAG), Exploitation in Ireland's Restaurant Industry, Dublin, décembre 2008, p. 9.

20. Une enquête montre que les migrants sont, dans l'ensemble, victimes de discrimination sur leur lieu de travail deux fois plus que les Irlandais; AMRI 2010, p. 32.

21. Eamon O'Shea, Kieran Walsh, The Role of Migrant Health and Social Care Workers in Ageing Societies. The Irish Context, WP-ICSG-2008-08, p. 63-70 et 130-131. Martha Doyle, Virpi Timonen, "The different faces of care work: understanding the experiences of the multi-cultural workforce ", Ageing \&Society 29, CUP, 2009, p. 341-343.

22. MRCI's RWAG, décembre 2008, p. 5 et 22.

23. ESRI, Migrant'Experience of Racism and Discrimination in Ireland, novembre 2006, 65 p.

24. Ibid., p. 37 et 38.

25. Organisme indépendant qui a remplacé l'Employment Equality Agency en 1999. [http://www.equality.ielen/], consulté le 10 janvier 2015.

26. Philip O’Connell et Frances McGinnity, Immigrants at Work, ESRI, Dublin, 2008, p. 34-42. 
En ce qui concerne les salaires moyens des immigrantes par rapport à ceux des Irlandaises, les chiffres reflètent des inégalités marquées, variables selon les secteurs. À niveau d'emploi égal, elles gagnaient $14 \%$ de moins en moyenne que les Irlandaises en $2007^{27}$. On trouve les salaires les plus bas dans le secteur de la restauration et de l'hôtellerie, où la représentation syndicale est faible ${ }^{28}$. Pourtant les documents de référence soulignent que le niveau moyen d'études de l'ensemble des immigrants est supérieur à celui des autochtones de manière significative $^{29}$. Les migrantes, européennes ou non, notamment, sont employées à un niveau inférieur à celui auquel elles pourraient prétendre. Les pertes d'emplois consécutives à la crise économique ont affecté les migrantes davantage : le pourcentage est supérieur à celui des Irlandaises, respectivement de $11,2 \%$ et 7,8 \% en $2009^{30}$, ce qui indique une stabilité moindre de leur emploi.

De façon générale, les migrants des douze nouveaux États membres, depuis les élargissements de 2004 et 2007, se trouvent au bas de l'échelle dans la hiérarchie de l'entreprise. Cela est le cas dans les commerces de gros et de détail où les Européens des anciens états membres accèdent à un niveau de responsabilités supérieures, à l'instar des autochtones ${ }^{31}$. Les $120^{32}$ fermes à champignons constituent un secteur spécifique où s'illustrent l'ensemble des problèmes pour les migrants d'Europe de l'Est ${ }^{33}$. En 2006, environ $3000^{34}$ personnes y sont employées à la culture elle-même, des femmes en majorité, originaires notamment des pays baltes. Ces migrantes effectuent la cueillette en particulier. Le taux horaire est de 5 à $6 €$ en moyenne, alors qu'il devrait être d'un peu plus de $8 €$. Les cueilleuses sont d'ailleurs payées au kilo ou à la quantité de champignons cueillis, mode de calcul qui les désavantage. Les droits aux assurances sociales, aux congés hebdomadaires et annuels, aux jours fériés, ne sont pas respectés; la protection de la santé pour ces personnes exposées à des substances chimiques, n'est pas assurée, notamment dans le cas des femmes enceintes; de plus, les mesures de sécurité sont insuffisantes et les conditions d'hébergement mauvaises ${ }^{35}$.

L'économie qualifiée de grise, parce qu'elle échappe facilement à la réglementation en vigueur, est un autre secteur d'emploi pour la main-d'œuvre féminine étrangère, venue de partout, où les pratiques discriminatoires sont fréquentes. Les migrantes y sont aides à domicile chargées des soins aux enfants ou aux per-

27. O'Connell et McGinnity, op. cit., 2008, p. 14.

28. CSO, National Employment Survey 2007, Dublin, July 2009, p. 43 et 53.

29. $31 \%$ env. d'immigrants, $27 \%$ env. d'autochtones. CSO, Census 2011, Profile 6 Migration and Diversity, p. 24.

30. AMRI, op. cit., 2010, p. 17.

31. Ibid., p. 42.

32. Chiffres pour 2006.

33. MRCI, The Mushroom Workers Support Group (MWSG), Harvesting Justice, Mushroom workers call for change, Nov. 2006. La main d'œuvre recrutée vient aussi d'Ukraine, Moldavie, Chine, Thaïlande, p. 8-11.

34. MRCI, MWSG, op. cit., 2006, p. 8.

35. Ibid., p. 14-17. 
sonnes âgées. Ce type d'emploi s'est développé, en Irlande, avec la croissance économique des années 1990 qui a incité nombre d'Irlandaises diplômées à sortir de leur rôle traditionnel pour faire carrière. Dans l'économie grise, les contrats de travail, quand il y en a, sont rarement respectés, les travailleuses domestiques étant souvent payées de la main à la main ${ }^{36}$. Les abus sont difficiles à réprimer car l'inspection du travail a difficilement accès au domicile familial ${ }^{37}$. En outre, avant 2004, l'Employment Equality Act de 1998 ne s'appliquait pas à la sphère privée.

Selon une optique féministe, il apparaît donc que les migrantes dans le domaine de l'emploi sont objets de discrimination à plusieurs niveaux. Elles le sont comme femmes reléguées à des emplois subalternes, selon la division traditionnelle des rôles. Comme catégorie sociale vulnérable, nombre d'immigrantes sont exploitées, parfois maltraitées, par des Irlandaises dont la condition sociale est meilleure. Bien que celles-ci soient également assignées aux responsabilités familiales et domestiques, elles sont en position de déléguer les tâches quotidiennes à de la main-d'œuvre féminine étrangère moins favorisée. Et, finalement, comme employées, les migrantes sont soumises à la législation pesante du pays dit d'accueil, au mépris de leur niveau de qualifications souvent, afin de pourvoir aux besoins de membres de leur famille restés dans le pays d'origine ${ }^{38}$.

L'analyse de la condition dans l'emploi des immigrantes en Irlande fait ainsi apparaître des injustices tant par le statut qui leur est accordé, le salaire qui leur est attribué, que par le traitement de nature raciste qu'elles peuvent endurer personnellement. Les victimes sont surtout les immigrantes africaines et d'Asie du SudEst. Il convient cependant de ne pas tomber dans une généralisation abusive car les constats se fondent sur des enquêtes qualitatives, portant sur un nombre limité de cas. La situation des employées venant d'Europe de l'Est est ainsi préférable : à la différence des migrantes hors Europe, elles ne sont pas soumises au système du permis de travail ${ }^{39}$, qui place la main-d'œuvre étrangère sous la coupe de l'employeur, dont ces personnes dépendent pour le renouvellement de leur contrat ${ }^{40}$. Leurs possibilités de changer d'emploi sont également entravées. Mais ce sont les migrantes recrutées dans l'économie grise de la sphère privée, qui, plus que les autres encore, sont exposées à la violation de leurs droits, phénomène qui se développe à l'échelle mondiale ${ }^{41}$.

36. Salaire net hebdomadaire $250 €$ en moyenne, MRCI, Private Homes, a Public Concern, 2004, p. 14.

37. MRCI, Private Homes..., 2004, p. 17. Ce rapport offre une perspective documentée sur ce problème en Irlande : les domestiques en Irlande viennent d'Asie : des Philippines surtout, d'Europe de l'Est, d'Afrique.

38. Martha Doyle, Virpi Timonen, in Ageing \& Society 29, CUP, 2009, p. 337-350.

39. AMRI, op. cit., 2010, p. 19; Barry, op. cit., 2010, p. 6-7.

40. Doyle et Timonen, op. cit., 2009, p. 346-347.

41. "Cent millions de "domestiques" dans le monde ", Le Monde, 10 janv. 2013, p. 7 et "Deux visages du Bangladesh ", 3-4 fév. 2013, p. 6. 


\section{Aliénation}

Des réformes successives ont voulu mettre en résonance la loi du pays et son économie en voie de globalisation. Mais ont-elles permis l'insertion des étrangers dans la société du pays? Une distinction entre les citoyens irlandais et les ressortissants d'autres pays a été établie, et reconduite, à travers diverses formules contenues dans les textes. Ces différentiations, qui sont allées en se complexifiant, ont néanmoins reposé sur la distinction binaire entre les citoyens irlandais et les autres $^{42}$, dont les statuts diffèrent selon qu'ils sont immigrants économiques ou politiques, qu'ils proviennent de la zone Europe ou d'ailleurs, point étudié par l'universitaire Bryan Fanning dans un ouvrage publié en $2007^{43}$. Se documenter sur les femmes migrantes, à l'intérieur de ces catégories, c'est se heurter au manque de statistiques de genre. Il n'en demeure pas moins que l'ensemble des dispositions légales sont fondées sur une division entre les autochtones et les nonautochtones, car il s'agit, selon le sociologue Steven Loyal, de préserver une homogénéité ethnique et culturelle irlandaise. Dans un ouvrage de 2011, il dénonce la bureaucratie irlandaise, et, à travers cela, la propension des autorités du pays à instaurer des classifications rigides, qui sont autant de lignes de séparation entre ceux qui sont citoyens irlandais et ceux qui ne le sont pas, à leur tour soumis à des subdivisions, dans le but, selon Steven Loyal, d'asseoir la domination, le contrôle de l'État tout en répondant aux besoins de l'économie libérale ${ }^{44}$. Comment donc traiter l'objectif paradoxal de développer le pays par l'ouverture de ses frontières, et de le protéger de l'influence de l'autre venu d'ailleurs? L'universitaire Ursula Barry décrit une politique migratoire hautement régulée et restreinte ${ }^{45}$, qui tend actuellement à attirer dans le pays les seuls migrants des pays hors Espace Economique Européen hautement qualifiés, à travers le système de la carte verte ${ }^{46}$. Les ressortissants des nouveaux États-membres depuis 2004 se dirigent, eux, vers des emplois plus modestes dans les services ${ }^{47}$.

Le système de permis de travail ${ }^{48}$ s'applique aux migrants économiques, candidats à des emplois non pourvus par les migrants européens, c'est-à-dire à ceux qui sont extérieurs à l'EEE, ainsi qu'aux Roumains et Bulgares, pour qui l'accès au marché du travail irlandais a été restreint pendant 5 ans après l'accession,

42. Citizens/aliens, selon l'Aliens Act de 1935; nationals/non nationals selon le Nationality and Citizenship Act de 1956. A partir de l'adhésion de l'Irlande à l'UE, de nouvelles subdivisions sont apparues, distinguant les citoyens des pays membres, des autres citoyens. Loyal, 2011, p. 43.

43. Bryan Fanning (ed.), Immigration and Social Change in the Republic of Ireland, Manchester UP, 2007, p. 6-26.

44. Loyal, op. cit., 2011, chap.3, p. 56.

45. Ursula Barry, op. cit., octobre 2010, p. 2.

46. 47400 immigrants de cette catégorie en 2004, 23417 en 2009, AMRI, op. cit., 2013, p. 21.

47. Loyal, op. cit., 2011, p. 166.

48. Voir note 40. 
comme l'autorise une disposition européenne de 2004. Des travaux de recherche évoquent les abus de pouvoir, cautionnés par les autorités en quelque sorte ${ }^{49}$.

Quant aux demandeurs d'asile ${ }^{50}$ non autorisés à travailler et disposant de moyens matériels très faibles alloués par le gouvernement ${ }^{51}$, ils sont marginalisés de fait. Ils vivent ainsi en vase clos, dans des centres d'hébergement situés aux quatre coins du pays. La procédure administrative pour obtenir le statut de réfugié dure en général plusieurs années -7 ans dans quelques cas ${ }^{52}$ - même si le service en charge de ces dossiers évoque un délai ramené à quelques mois ${ }^{53}$. Les demandes satisfaites sont peu nombreuses : l'Irlande compte parmi les pays les plus réticents $^{54}$ à accorder ce statut. Les femmes seules qui ont leurs enfants avec elles, situation fréquente pour les Africaines notamment ${ }^{55}$, sont logées dans une ou deux pièces exiguës. Elles sont en proie à l'insécurité, souffrent de n'avoir aucune intimité, sont désœuvrées, et en perte de repères, puisqu'elles ne peuvent pas même accomplir les tâches quotidiennes telle que la préparation des repas, comme l'impose le régime de prise en charge directe pour les demandeurs d'asile ${ }^{56}$.

Comme la gestion de la question de l'immigration mêle de façon étroite les droits des personnes dans la société, et leur statut dans le monde de l'emploi, les demandeurs d'asile, à l'instar de la plupart des immigrants économiques, vivent dans des conditions précaires, et sont aux prises avec des formalités administratives et judiciaires vétilleuses. Steven Loyal voit comme du cynisme le fait que l'on privilégie l'immigration de nouveaux venus de couleur et culture proches de celles des autochtones, attirés par l'emploi seulement et n'envisageant pas de s'établir définitivement en Irlande ${ }^{57}$.

Les diverses catégories de femmes migrantes sont, quant à elles, plongées dans la société patriarcale du pays d'accueil où, aux discriminations traditionnelles de genre, s'ajoutent des formes d'aliénation spécifiques entraînées par la législation complexe en place. La question du regroupement familial en est un exemple. Autorisé pour les réfugiés, le regroupement familial, pour les autres personnes, dépend d'une part du statut de celui qui demande ce regroupement,

49. Kieran Allen, «Neo-liberalism and immigration » dans Bryan Fanning (ed.), op. cit., 2007, p. 85-98.

50. Leur nombre global décroit depuis le début de la crise : de 4000 env. en 2007, à env. 950 en 2012; [http://www. orac.ie/website/oracloracwebsite.nsflpage/orac-stats], consulté le 19 juin 2014. Mais la proportion de femmes est passée de 1/3 en 2006 (Pillinger, op. cit., 2007, p. 18) à 3/5 en 2012 (Office of the Refugee Applications Commissioner (ORAC), Annual Report 2012, p. 15).

51. Environ $20 €$ hebdomadaires pour un adulte, $10 €$ pour un enfant selon le régime de direct provision, AMRI, p. 11, Barry, op. cit., 2010, p. 7.

52. AMRI, op. cit., 2010, p. 11; AMRI, op. cit., 2013, p. 11.

53. ORAC, Annual report 2011, p. 3.

54. [http://www.irishrefugeecouncil.ie/] consulté le 20 juin 2014.

55. Les Nigérianes sont les plus nombreuses (Fanning 2011, p. 72). Elles représentent aussi la forte proportion de parents isolés, CSO, Press statement, Census 2011 Profile 6 Migration and Diversity, p. 16.

56. AMRI, op. cit., 2010, p. 11.

57. Loyal, op. cit., 2011, p. 173 et également chapitre 9, p. 154-187. 
d'autre part de ses liens familiaux avec les bénéficiaires potentiels de la mesure en provenance de pays extérieurs à l'Europe ${ }^{58}$. En bref, la législation avantage les demandeurs dont le statut et la situation socioprofessionnelle sont stables et favorables. Alors que le processus de réunification est accéléré dans le cas où les demandeurs sont citoyens irlandais ou des personnes de catégories socioprofessionnelles supérieures, il est ralenti pour les autres ${ }^{59}$. Cela constitue un problème pour les femmes, qui, de plus en plus, émigrent seules. Une enquête qualitative, faite auprès d'infirmières indiennes et philippines, révèle que le regroupement familial est la condition sine qua non pour qu'elles décident de rester dans le pays. Elles dénoncent la frilosité et la complexité du système ${ }^{60}$. Lorsque le conjoint (la conjointe) bénéficie du regroupement familial, il (ou elle) est confronté à la difficulté de trouver du travail, car la législation irlandaise, certes l'autorise à avoir un emploi, mais cela est encadré par la législation applicable à tout migrant, en ce qui concerne le permis de travail notamment ${ }^{61}$.

Les conjointes de migrants forment une catégorie sociale de femmes au foyer, dépourvues de moyens linguistiques ou matériels, sans autonomie ni capacité de s'aider elles-mêmes en cas de problèmes tels que la violence domestique ${ }^{62}$. Les migrantes dépendantes victimes d'abus ne peuvent quitter leur conjoint, car leur présence légale dans le pays est liée à leur statut d'épouse. Elles se retrouvent ainsi dans une situation d'aliénation extrême, déterritorialisées à la fois dans la sphère privée et publique, et doivent de plus justifier de cinq années dans le monde de l'emploi pour obtenir une carte de séjour ou la naturalisation. La tentation de se tourner vers des emplois non déclarés ${ }^{63}$ peut alors être forte.

Les obstacles pour s'installer dans le pays se sont accrus avec le référendum de 2004 qui a durci les conditions d'obtention de la nationalitét ${ }^{64}$. Des organisations spécifiques se battent pour déverrouiller le système. La réforme de $2010^{65}$, non promulguée, lacunaire à propos du regroupement familial, restrictive en ce qui concerne l'accès à l'emploi des conjoints dépendants, a déçu les espoirs d'organisations telles que l'Immigrant Council of Ireland.

À ce tableau sombre s'ajoute l'institutional discrimination dont les conséquences peuvent être lourdes pour les migrants, car les dispositions sur l'immigration sont régies par des mesures administratives plutôt que par la loi. Les femmes migrantes,

58. [http://www.nascireland.org//wp-content/uploads/2013/09/Family-Reunification-Factsheet-2.pdf], consultés le 11 janv. 2015.

59. [http://www.justice.ielen/JELR/Pages/PB13000447], p. 7, consulté le 11 janvier 2015.

60. Niamh Humphries et al., op. cit., Aug. 2009, p. 4.

61. Pillinger, dans Barry (ed.), op. cit., 2008, p. 198.

62. ESRI, Migrants' Experience of Racism and Discrimination in Ireland, Dublin, Nov. 2006, p. 18 et 23.

63. Pillinger dans Barry (ed.), op. cit., 2008, p. 198-199.

64. Bairbre Ni Chiosain, "Passports for the New Irish? The 2004 citizenship referendum ", Études Irlandaises, Automne 2007, $\mathrm{n}^{\circ} 32.2$, p. 31-47.

65. Immigration, Residence and Protection Bill, 2010. 
plus que les hommes semble-t-il, sont victimes de cette discrimination institutionnelle dans les divers bureaux et services, comme le révèlent les résultats d'une enquête effectuée en 2005. Des exemples concrets de rebuffades, de difficultés rencontrées dans les contacts avec les agents, sont relatés par le menu dans un ouvrage de Steven Loyal ${ }^{66}$. Le critère de résidence continue dans le pays détermine l'octroi de Habitual Residency Condition qui ouvre les droits aux avantages sociaux ${ }^{67}$. Lagent administratif effectue le calcul crucial de cette durée de résidence continue, la notion de continuité n'étant pas exempte d'ambiguïté. Les migrantes à qui l'HRC est refusée, de façon contestable parfois, risquent la marginalisation ${ }^{68}$.

\section{Mobilisation}

L'Irlande a, certes, adopté une politique frileuse vis-à-vis des étrangers ayant l'intention s'installer dans le pays, mais se mobilise pour lutter contre les réseaux de trafiquants internationaux qui sévissent sur son sol $^{69}$. En cela les autorités sont en phase avec la société : le mouvement pour "éteindre les lumières rouges ", Turn Off The Red-Light Campaign (TORL), s’y est peu à peu constitué à partir de 2006 et a mis en relief les liens étroits entre la prostitution et l'immigration, souvent forcée, de femmes. Des travaux de recherche menés à partir de 2007 montrent que plus de $90 \%$ des personnes qui s'adonnent à ce commerce en appartement, par le biais d'agences sur la Toile, sont des migrantes ${ }^{70}$. Il ne fait pas de doute que les flux migratoires, depuis le milieu des années 90, se sont accompagnés de trafic, de mineures parfois, facilité et banalisé par le tissage d'organisations opérant sur internet, sous l'appellation politiquement correcte d'escort agencies. TORL vise à combattre le fléau par la criminalisation des clients, politique à l'étude en France et d'ores et déjà appliquée en Suède, Norvège, et Islande.

Le site de TORL montre qu'en deux ans, de 2011 à 2013, une soixantaine d'organisations $^{71}$ ont rejoint la campagne. L'ensemble fédère des organisations de femmes $^{72}$, des syndicats (ICTU ${ }^{73}$, SIPTU), des agences d'aide aux migrants, dont

66. ESRI, Migrants' experience..., op. cit., novembre 2006, p. viI, 51-52 et 60; Loyal, 2011, op. cit., p. 50-56.

67. Habitual Residency Condition HRC, qui fixe à 2 ans, depuis 2004, la durée de résidence continue requise pour prétendre, en tant qu'immigrant, aux prestations sociales. Fanning 2011, p. 134.

68. M.J. Da Col Richert, "Women of Ireland, from economic prosperity to austere times: who cares? ", Etudes Irlandaises, automne-hiver 2012, Vol. 37, n² 2, p. 19-31. G; Demetrios et al., Migration and Immigrants..., Migration Policy Institute, Washington DC 2010, p. 91-93. AMRI, op. cit., 2010, p. 9.

69. [http://www.dublinpact.ieldignity/PDF/Dignity\%20Exec\%20fr.pdf], p. 4; consulté le 14 janvier 2015.

70. ICI, Globalisation, Sex Trafficking and Prostitution, Dublin, 2009, p. 9.

71. Liste sur le site [www.turnofftheredlight.ie].

72. [www.nwci.ie], consulté le 25 juin 2014.

73. L'Irish Confederation of Trades Unions, qui a refusé de s'associer à la création d'un syndicat de Travailleurs du Sexe durant l'été 2009; [http://en.wikipedia.org/wiki/Prostitution_in_the_Republic_of_Ireland], consulté le 20 oct. 2013. 
l'ICI, des communautés religieuses. TORL entend influencer les pouvoirs publics, grâce notamment à l'appui du parti travailliste, membre de la coalition gouvernementale actuelle.

L'Irlande s'est aussi associée au Dignity Project ${ }^{74}$, au tournant des années 2009-2010. Cette initiative européenne ${ }^{75}$ procure un financement aux partenaires du projet, agences gouvernementales et $\mathrm{ONG}^{76}$. La terminologie Dignity Project, évocatrice de rectitude morale, est en résonance avec la campagne qui "veut éteindre les lumières rouges ", vues comme signes de dépravation signalant les maisons closes et les quartiers voués à la prostitution.

D'un point de vue féministe, la prostitution est un enjeu controversé. Tandis que certains courants ${ }^{77}$ revendiquent la liberté pour les femmes de disposer de leur corps, la campagne irlandaise s'inscrit dans une perspective contraire. Elle est abolitionniste afin, surtout, de contrecarrer le développement de la délinquance liée au trafic, en regard de quoi tolérer la prostitution au nom de la liberté individuelle pèse peu. Le féminisme abolitionniste renvoie la prostitution à des rapports sociaux de genres inégalitaires, à une forme de domination masculine. Il est aussi qualifié de puritain ${ }^{78}$, et fait écho à la lutte contre la prostitution, menée au début du siècle précédent par des militantes prônant l'austérité en matière de moralité sexuelle. La cause abolitionniste s'oppose aussi à ceux et celles qui sont favorables à la légalisation de la prostitution, et revendiquent un statut d'employées pour les personnes impliquées dans ce qu'ils désignent comme un secteur d'activitét ${ }^{79}$. Wendy Lyon, et d'autres, de Feminist Ire ${ }^{80}$, défendent une position légaliste, rejettent ainsi ce qu'elles décrivent comme "your fluffy feminism ", féminisme " en peluche ", angélique, aveugle à la rugosité des réalités humaines.

Comment les organisations de migrantes se sont-elles positionnées par rapport à la question abolitionniste? Une seule de ces organisations, semble-t-il, figure dans la liste des participants à la campagne. Il s'agit d'AkiDwA ${ }^{81}$, organisation de migrantes africaines à l'origine, mise sur pied de 1999 à 2001, affiliée au NWCI, qui œuvre à l'intégration des immigrantes dans la sphère publique en général ${ }^{82}$. AkiDwA a acquis une visibilité particulière, liée sans doute au niveau d'études de

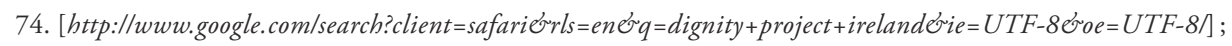
[http://www.dublinpact.ieldignity/PDF/Finalevaluationcover_merged.pdf]; consulté le 14 janv. 2015.

75. L'Espagne, la Lithuanie, le Royaume-Uni (Écosse surtout).

76. [http://www.dublinpact.ieldignity/PDF/Finalevaluationcover_merged.pdf]; consulté le 14 janv. 2015.

77. Ainsi, Elisabeth Badinter ou la juriste Marcella Iacub, en France.

78. Nathalie Heinich, «Une loi culpabilisante contre le féminisme puritain », Le Monde, 8 novembre 2013, p. 18.

79. La Sex Workers Alliance a été formée en 2009.

80. [http://www.irishleftreview.org/author/wendy-lyon/] consulté le 20 oct. 2013. [http://feministire.wordpress.com/ who-we-arel, consulté le 20 oct. 2013.

81. Akina Dada Wa Africa, Sisters of Africa en swahili.

82. Thème du séminaire organisé par Akidwa et l'ICI, 28 juin 2013 : « Migrant women need choices, not exploitation ". 
nombre de migrantes hors Europe. Un fascicule publié par AkiDwA en 2011 s'intitule Home and Away, Migrant Women Transforming Ireland. On y met en relief une optique féministe, un engagement contre la violence et la discrimination de genre. L'universitaire Ronit Lentin, membre de l'organe consultatif de l'organisation $^{83}$, souligne son action politique ${ }^{84}$. Pourtant, même si AkiDwA a ouvert le féminisme irlandais à de nouveaux enjeux, les liens ne se sont pas tissés avec les organisations de femmes autochtones.

TORL est néanmoins un lieu de convergence où une autre organisation de femmes, Ruhama ${ }^{85}$, s'emploie à réinsérer dans la société les femmes marginalisées et les victimes de trafic. Ruhama, créée en 1989, a pris acte de l'interaction entre le nouveau visage de la prostitution en Irlande et l'immigration depuis une quinzaine d'années, et met en évidence la vulnérabilité extrême des migrantes en attente du statut de réfugiées, ou d'un permis de séjour ou de travail dans le cas de celles qui ont été victimes de réseaux et veulent légaliser leur situation. Ruhama enracine son action à la fois dans le discours féministe abolitionniste et dans la morale chrétienne. Un état des lieux sur la question de la prostitution en Irlande, auquel a collaboré Ruhama, a d'ailleurs été financé par les Sœurs de la Charitée ${ }^{86}$. Ruhama occupe donc une position charnière, fédératrice, qui tient compte des évolutions de la société irlandaise et s'ouvre aux nouveaux enjeux concernant les populations féminines dans leur diversité.

Les migrantes arrivées en Irlande pendant le Tigre celtique sont dans l'ensemble en situation d'infériorité, et cela est en lien avec la nationalité, la classe sociale, le genre où s'enracinent des handicaps qui se superposent. Elles sont majoritairement recrutées dans des secteurs d'emplois féminisés : les soins, les travaux domestiques, la grande distribution, la restauration et l'hôtellerie. Les qualificatifs 3C cleaning, caring, catering, que l'on pourrait traduire en français par les $3 \mathrm{~N}$ : nettoyer, nourrir, soigner, sont associés à ces emplois. De façon plus amère, ils se rangent aussi dans la catégorie $3 \mathrm{D}$, dirty, dangerous ${ }^{87}$, demeaning, dont l'équivalent en français pourrait être $3 \mathrm{~S}$ : sale, subalterne, sous-payé. La législation irlandaise, restrictive en termes d'accès aux aides sociales et au système de santé, laisse aussi place à l'arbitraire administratif dans le traitement des dossiers déposés par ceux qui veulent s'installer dans le pays. Les migrantes souffrent particulièrement

83. Ronit Lentin (2009) "Migrant women's networking: New articulations of transnational ethnicity ", dans G. Bhattacharyya (ed.), Ethnicities and Values in a Changing World, Farnham and Burlington VT, Ashgate Publications, p. 65-82.

84. Notamment, après le référendum de 2005 sur la citoyenneté, R. Lentin, op. cit., 2009, p. 7.

85. Ruhama signifie renewed life en hébreu.

86. Globalisation, Sex Trafficking and Prostitution..., ICI en collaboration avec Women's Health Project, Ruhama, 2009.

87. Le terme " dangereux " évoque a priori plutôt les emplois masculins dans la construction, mais aussi les emplois et activités dans l'économie grise ou illégale occupés par les migrantes et sont, de ce fait même, dangereux. 
du manque de dispositions en faveur de la réunification familiale. Celles qui arrivent en qualité de conjointe peinent à sortir de la dépendance ou de la sphère privée transformée parfois en carcan. Pourtant, l'Irlande est parvenue à absorber sans violence, en un peu plus d'une décennie, l'arrivée massive de migrants avec l'appui d'organisations non gouvernementales qui œuvrent en faveur des étrangers dans le pays. Les migrantes créent leurs propres organisations dans le but de se faire une place dans la société, qu'elles disent vouloir transformer, et bénéficient de relais dans les milieux universitaire et syndical, dans certains partis politiques. Un courant féministe imprégné de valeurs morales catholiques, allié à nombre d'acteurs de la société irlandaise, se mobilise depuis quelques années afin d'abolir la prostitution et le trafic liés à l'immigration. Nombre de femmes marginalisées seront alors libérées de cette menace, mais cesseront-elles pour autant d'être ghettoïsées? 